\title{
The experience of giving up smoking was described as encompassing 7 major categories
}

\author{
Cobb AK, Bott MJ, O'Connell KA. A qualitative/interpretive taxonomy of stop smoking strategies (QU/ITS). West J Nurs Res 1997 \\ Dec;19:702-25.
}

\section{Question}

What words and concepts are used by people to describe their coping strategies within the first 10 days after giving up smoking?

\section{Design}

A taxonomy inductively derived from narrative accounts.

\section{Setting}

A large midwestern city in the US.

\section{Participants}

36 participants (mean age $41 \mathrm{y}, 58 \%$ women) who were recruited from smoking cessation programmes. The mean number of attempts to stop smoking was 4.7. Participants smoked a mean number of 24 cigarettes each day and had begun smoking at a mean age of 17 years.

\section{Methods}

Participants were randomly assigned to 5 time blocks of 3 consecutive days within the first 10 days after they had stopped smoking. Participants recorded thoughts, feelings, and actions for each coping episode as they occurred using a hand held tape recorder. A coping episode consisted of any thought or behaviour that was used to avoid smoking or to reduce or prevent the urge to smoke. The NUD•IST software programme was used to analyse data and to construct a taxonomy. The completed taxonomy was validated by using a focus group which comprised 3 of the study participants.

\begin{abstract}
Main results
477 coping episodes were tape recorded. 7 major taxons reflected the process of dealing with the urge to smoke: (1) social context, which had 5 categories: factual details (place, time of day, companions, activities, and availability of cigarettes); relationships; vulnerabilities; triggers that increased the urge to smoke; and external constraints such as a no smoking rule in places that participants visited frequently; (2) anticipatory efforts, which included plans to avoid triggers for smoking, plans for activities to combat the urge to smoke, and plans for physical replacements for cigarettes; (3) levels of awareness, which included an awareness of the intensity of the urge to smoke, attempts not to think about cigarettes, thoughts about cigarettes, and need for a physical replacement; (4) urges, which were described in terms of their intensity, duration, and frequency; (5) coping strategies, which included thoughts, activities, breaking a habitual routine, the use of a no smoking rule, and waiting it out; (6) effects, which included physical and emotional responses and the evaluation of the coping strategies in terms of their effectiveness, physical effects, and emotional effects; and (7) the metaphors used for cigarettes and for the process of coping with smoking cessation.
\end{abstract}

\section{Conclusion}

The process of smoking cessation was described by study participants as encompassing 7 major taxons: the social context for urges and coping strategies, anticipatory efforts, levels of awareness, urges to smoke, coping strategies, effects of coping strategies, and metaphors.

Source of funding: National Institute of Nursing Research.

For article reprint: Dr A K Cobb, School of Nursing, University of Kansas at Kansas City, 3901 Rainbow Boulevard, Kansas City, KS 66160-7501, USA. Fax +1913 5881660.

\section{Commentary}

Cigarette smoking continues to be the single, most preventable cause of mortality and morbidity in the US. ${ }^{1}$ Although many smokers realise the health consequences and make attempts to stop, relapse rates among those who try to stop smoking average as high as $80 \% .{ }^{2}$ Previous research has shown that using a coping strategy helps to resist smoking during a tempting situation; ${ }^{3}$ however, little is known about the nature of coping during smoking cessation. Cobb et al return to the source (ie, smokers) and ask them to describe what they felt, did, or thought to help them avoid smoking, reduce the urge to smoke, or prevent the urge to smoke. This first level inquiry identifies factors that are inherent in the experience of giving up smoking within the first 10 days of stopping-the time that is perhaps the most challenging for those attempting to stop.
The strengths of this study include the collection of qualitative data at the time the coping episode occurred ("real time") directly from the person attempting to stop. This is an improvement over previous research in which data were collected retrospectively using structured questions. The study participants were mainly white, middle aged, and well educated and the findings therefore may not reflect the experiences of the general population.

These data can help nurses to understand better the complexity of the giving up process. The experiences of those who stop smoking are multiple and varied. Awareness of the 7 taxons identified in this study may help nurses to collect more specific data about the coping strategies used by individual clients, to provide anticipatory guidance and support to clients in relation to coping strategies, and to design and evaluate interventions for helping individuals to stop smoking, including self help materials.

Nancy Nightingale Gillespie, RN, PhD Graduate Director and Associate Professor Department of Nursing University of Saint Francis Fort Wayne, Indiana, USA

1 Anderson CB, Wetter DW. Behavioral and pharmacological approaches to smoking cessation. Cancer Metastasis Rev 1997;16:393404.

2 United States Department of Health and Human Services. The health benefits of smoking cessation. Rockville, MD: Office of Smoking and Health;1990.

3 Baer JS, Kamarck T, Lichtenstein E, et al. Prediction of smoking relapse: analyses of temptations and transgression after initial cessation. I Consult Clin Psychol 1989;57: $623-7$. 